\title{
Differentiation of Human Dermal Mesenchymal Stem Cells into Cardiomyocytes by Treatment with 5-Azacytidine: Concept for Regenerative Therapy in Myocardial Infarction
}

\author{
Pravin D. Potdar and Preeti Prasannan \\ Department of Molecular Medicine \& Biology, Jaslok Hospital \& Research Centre, 15 Dr. G. Deshmukh Marg, \\ Mumbai, 400026 Maharashtra, India \\ Correspondence should be addressed to Pravin D. Potdar; ppotdar@jaslokhospital.net
}

Received 31 January 2013; Accepted 7 March 2013

Academic Editors: F. Fagioli and I. Rich

Copyright (C) 2013 P. D. Potdar and P. Prasannan. This is an open access article distributed under the Creative Commons Attribution License, which permits unrestricted use, distribution, and reproduction in any medium, provided the original work is properly cited.

Myocardial infarction (MI) is the leading cause of death worldwide. Stem cells regenerative medicine offers a promising approach to cure such degenerative disorders. Mesenchymal stem cells are thought to be one of the important types of stem cells which can differentiate into various lineages such as neuron, hepatocytes, and cardiomyocytes. In the present study, human dermal mesenchymal stem cells (hDMSCs) have been developed from human scalp punch biopsy and characterized for their mesenchymal phenotype so that these cells can be useful for differentiating into cardiomyocytes. 5-Azacytidine induces cardiomyocyte differentiation in vitro and therefore it has been used to differentiate hDMSCs cells into cardiomyocytes. It was observed that hDMSCs differentiated into cardiomyocyte within a period of 4 days to 15 days after treatment with $10 \mu \mathrm{M}$ and $20 \mu \mathrm{M}$ of 5 azacytidine. The cardiomyocyte phenotype was confirmed by studying expression of $\alpha$-cardiac actin, $\beta$-myosin heavy chain, and cardiac troponin T. Thus, this paper describes the differentiation of hDMSCs into cardiomyocytes which can be further be used for treatment of MI. This type of cell-based cardiac therapy will offer a new hope for millions of patients worldwide who are suffering from heart disease.

\section{Introduction}

Myocardial Infarction (MI) is most commonly known as heart attack. It is one of the leading causes of mortality all over the world mainly due to constant change in lifestyle with ever increasing stress. MI is not only seen among the 50-60-yearage group but presently has also become a common reason for death in the younger generation. MI is caused due to shortage of blood supply to a part of the heart which reduces the oxygen supply to the affected area leading to necrosis of the tissue (scar formation) and there is no complete recovery after such damage in adult humans [1]. For several decades, heart transplantation has been an option for therapy, but due to complications arising from host-graft rejection reaction and shortage of healthy donors, there is an immediate need to come up with other feasible options for the therapy of MI.

Recent development in stem cell research therapy and cell transplantation delivers great promise for the treatment of cardiovascular disorders $[2,3]$. Stem cells show immense promise for their application in medical field due to their unique ability to self-renew along with the potential to differentiate into different cell types. Such remarkable properties of stem cells show great promise in the field of regenerative medicine [4].

Mesenchymal stem cells are a population of adult multipotent unspecialized cells which can be isolated, cultured, expanded in vitro, and differentiated into various lineages. Earlier bone marrow was considered to be the major source of MSCs [5]. Recent studies have investigated various parts from the human body such as umbilical cord, cord blood, dental pulp, adipose tissue, and peripheral blood, tumour and can be used for development of MSCs [6-12].

In earlier days, a general concept regarding MSCs was that their differentiation is restricted only to few cell types such as adipocytes, chondrocytes, and osteocytes [13-15]. However, recent studies have proven the ability of MSCs to differentiate into cell types of all three lineages including cardiomyocytes [16-19]. The major advantage involved in utilising these MSCs for therapeutic purpose is their 
TABLE 1: Primer sequence for cardiac specific genes.

\begin{tabular}{|c|c|c|}
\hline Gene & Sequence & Base pair \\
\hline \multirow{2}{*}{$\alpha$-cardiac actin } & F: 5'TCTATGAGGGCTACGCTTTG3' & \multirow{2}{*}{259} \\
\hline & R: 5'GCCAATAGTGATGACTTGGC3' & \\
\hline \multirow{2}{*}{ Cardiac troponin $\mathrm{T}$} & F: $5^{\prime}$ AGAGCGGAAAAGTGGGAAGA3 ${ }^{\prime}$ & \multirow{2}{*}{234} \\
\hline & R: 5'CTGGTTATCGTTGATCCTGT3' & \\
\hline \multirow{2}{*}{$\beta$-myosin heavy chain } & F: 5'CGAGGCAAGCTCACCTACAC3' & \multirow{2}{*}{318} \\
\hline & R: 5'CATTAACAGCCTCCACGGCC3' & \\
\hline
\end{tabular}

Experiment 1-48 hours of 5-azacytidine treatment (cultured for 15 days)

$$
\text { Experiment 2-4 days of 5-azacytidine treatment }
$$

Experiment 3-8 days of 5-azacytidine treatment

FIGURE 1: It gives the complete outline of the different sets of experiments carried out. In experiment set $1 \mathrm{hDMSC}$ were treated with 5-azacytidine at concentrations of $10 \mu \mathrm{M}$ and $20 \mu \mathrm{M}$ for 48 hours followed by culture in normal growth media for 15 days. In the second and third set of experiments the hDMSCs cells were provided with media containing 5 -azacytidine $(10 \mu \mathrm{M}$ and $20 \mu \mathrm{M})$ for a period of 4 and 8 days, respectively.

remarkable immunosuppressive properties that can inhibit the proliferation and function of major immune cells including T cells, B cells, and NK cells [20].

Researchers have shown the differentiation of MSCs derived from sources like bone marrow into cardiomyocytes after treatment with 5-azacytidine, a chemical analogue of cytosine nucleoside [19, 21-23].

However, not much research has been carried out on differentiation of skin-derived MSCs into cardiomyocytes using 5-azacytidine. Our main objective is to find a minimally invasive and feasible source of human MSCs which can be readily available and utilized for treatment of various cardiovascular disorders. Skin occupies almost $90 \%$ of human body and thus availability of skin tissue for development of MSCs is the most advantageous in view of regenerative therapies. Recently, our laboratory has developed human dermal mesenchymal stem cells line (hDMSCs) derived from human-scalp punch biopsy from androgenic alopecia patient [24].

We have therefore proposed to use established hDMSCs line to differentiate into cardiomyocytes by treatment with 5 -azacytidine. The differentiation of skinMSCs cells into cardiomyocytes will be confirmed by using phase-contrast microscopy as well as by using cardiac specific markers such as $\alpha$-cardiac actin, $\beta$-myosin heavy chain, and cardiac Troponin T. The derived cells will be then beneficial for regenerative therapies in MI patients thereby giving hopes to millions of patients suffering from MI.

\section{Materials and Method}

2.1. Processing and Isolation of Mesenchymal Stem Cells from Human Scalp Biopsy. Scalp tissue punch biopsy was received from a female patient diagnosed with androgenic alopecia from Jaslok Hospital, with consent from the patient as per guidelines of the Ethical Committee of Jaslok Hospital and Research Centre. The mesenchymal stem cell line was developed from this tissue explant and designated as the human dermal mesenchymal stem cells (hDMSCs) [24]. Presently, this cell line is at passage 12 and is growing well. The hDMSCs were maintained in Dulbecco's modified medium (DMEM, HiMedia, India) supplemented with $10 \%$ fetal bovine serum (FBS, Gibco, Invitrogen), 1\% penicillin-streptomycin (HiMedia, India), $1 \mu \mathrm{l} / \mathrm{mL}$ insulin (Sigma, USA), and $2 \mu \mathrm{l} / \mathrm{mL}$ glutamine (HiMedia, India) as normal growth media. Cultured hDMSCs were maintained at $37^{\circ} \mathrm{C}$ with $5 \%$ carbon dioxide in a $\mathrm{CO}_{2}$ incubator (Thermo Scientific) and fresh media were fed every alternate day to these cells.

2.2. Growth Rate Analysis of Human Dermal Mesenchymal Stem Cell Line. The growth rate of hDMSCs was determined by MTT (3-(4,3-(4,5-dimethylthiazol-2-yl)-2,5diphenyltetrazolium bromide 5-dimethylthiazol-2-yl)-2, 5diphenyltetrazolium bromide) assay. MTT is reduced to formazan crystals in the mitochondria and this reaction is catalysed by the enzyme mitochondrial dehydrogenase. The formazan crystals absorb light at around $620 \mathrm{~nm}$ and thus provide a measurement of viable cells. The rate of cell proliferation was recorded by monitoring conversion of MTT to formazan crystals. The hDMSCs were counted using Neuber's haemocytometer and seeded at a density of $1 \times 10^{3}$ cells per well in a 96-well plate. This experiment was done in triplicates. Cells at the same density were seeded every day for the next 8 days. Cells were given complete growth media and media change was given every alternate day. On the 8th day, media was removed and replaced with $90 \mu \mathrm{l}$ of fresh media and $10 \mu \mathrm{l}$ of MTT (HiMedia, India) without any exposure to light and incubated for 4 hours at $37^{\circ} \mathrm{C}$ in $\mathrm{CO}_{2}$ incubator. After 4 hours, media was removed and replaced with dimethyl sulfoxide (DMSO, Merck) and absorbance was measured at $620 \mathrm{~nm}$ on microplate reader model SUNRISE (Tecan, India).

\subsection{Morphological Analysis Using Phase-Contrast and Light Microscopy}

2.3.1. Phase-Contrast Microscopy. The morphology of cultured cells was observed using phase-contrast microscope (Carl Zeiss Co.) which was attached to the computer having TSView software for observing and capturing the images. 


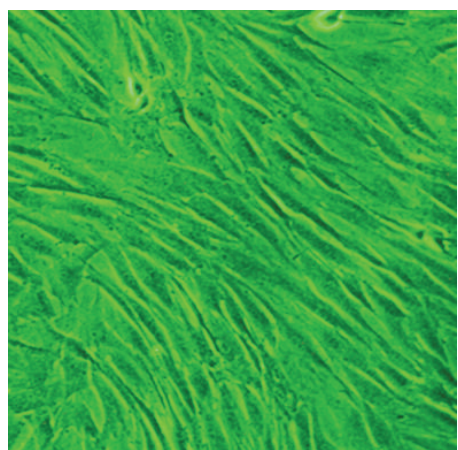

(a)

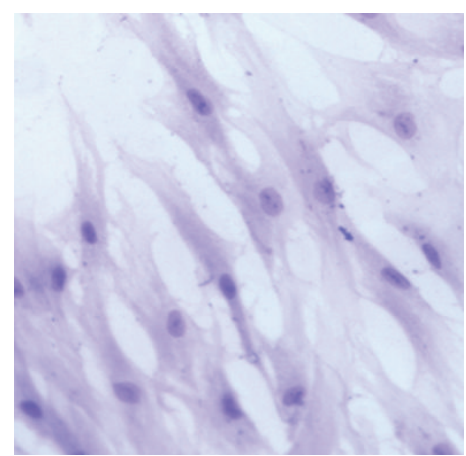

(b)

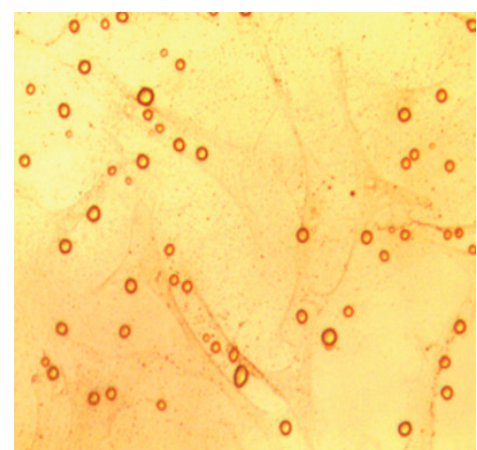

(c)

FIGURE 2: (a) shows the phase-contrast micrographs of confluent hDMSCs cell line at passage 12 . The cultures seem to be healthy and growing well. (b) represents the light microscope image of Giemsa stained hDMSCs. The cells have a distinct and round nucleus with slight cytoplasmic granules. (c) shows the light microscope images of hDMSCs stained with Oil Red O stain wherein the red stain was taken up by the oil secretions of the cell and not by the cell itself.

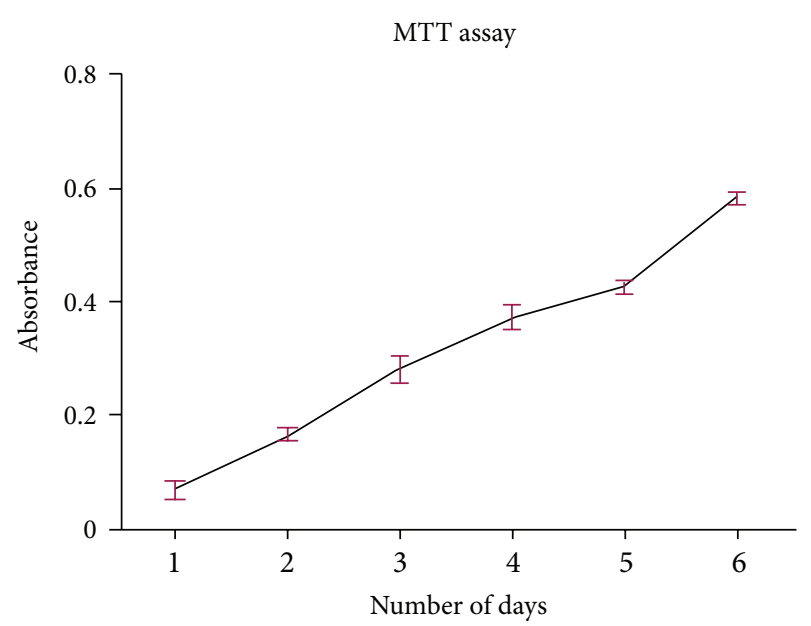

FIGURE 3: It shows bar graph indicating the growth rate of hDMSCs cell line. The standard deviation of MTT assay reading taken from day 1 to day 6 was plotted on a graph which shows the steady rise in the cell proliferation rate.

The cells were observed under 20x and 40x magnification. Cells were regularly monitored using phase-contrast microscope and images were captured for analysis.

2.3.2. Light Microscopy. For morphological analysis by light microscopy, hDMSCs were grown on sterile cover slips and maintained in normal growth media for 2 days till cells showed partial confluence prior to staining.

Giemsa staining was done to analyse the basic morphology of hDMSCs cell line. Approximately $1 \times 10^{4}$ cells were plated on sterile cover slips placed in $35 \mathrm{~mm}$ dishes (nunc) and fixed with 50\% methanol. Cells were then treated with filtered Giemsa Stain (Fisher Scientific) followed by washing with distilled water and observation under light microscope.

Oil Red O staining was performed to study the lipid nature of hDMSCs. For staining, approximately $1 \times 10^{4}$ cells were plated on sterile cover slips placed in $35 \mathrm{~mm}$ dishes

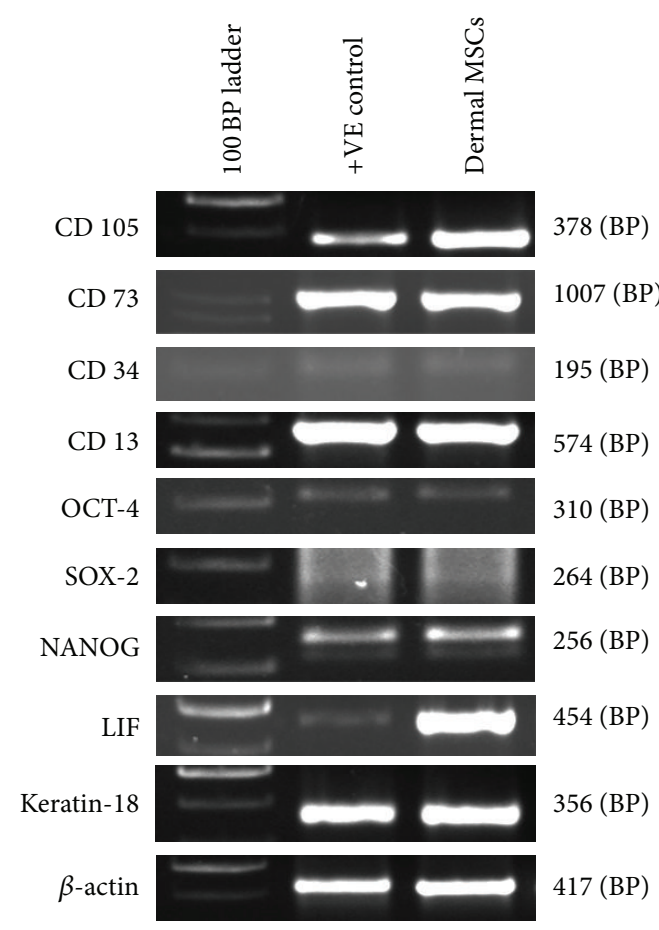

FIGURE 4: Characterization of hDMSCs cell line using molecular markers showing positive expression of CD105, CD73, CD13, CD44, Oct-4, SOX-2, NANOG, LIF, keratin-18, and $\beta$-actin.

(nunc) and fixed with 50\% methanol followed by washing with distilled water, and later these cells were treated with $60 \%$ isopropanol before treating with the Oil Red O stain (Sigma Aldrich) and observed under light microscope.

Alizarin Red staining was performed to study the changes in the basic protein structure of cells following 5-azacytidine treatment. For staining with Alizarin Red, and $1 \times 10^{4}$ hDMSCs cells were plated and treated for a period of 4 days with 5-azacytidine $(10 \mu \mathrm{M}$ and $20 \mu \mathrm{M})$ along with untreated control hDMSCs. On completion of 4 days, cells were fixed 


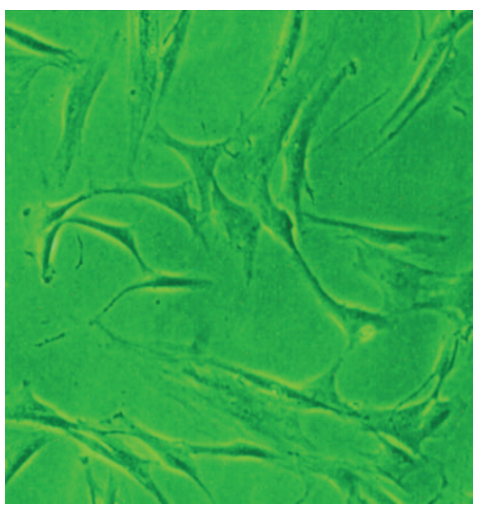

(a)

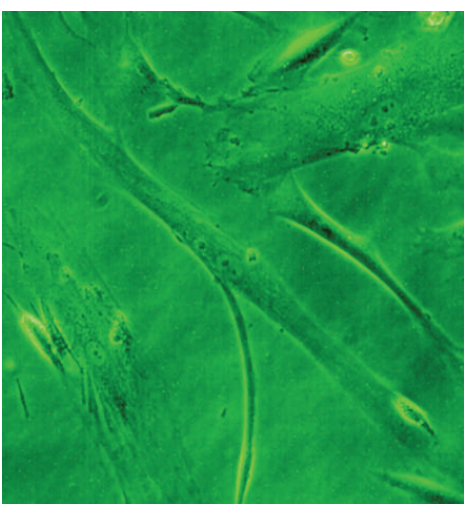

(b)

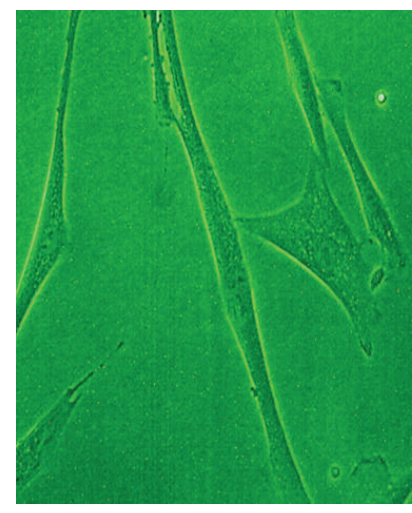

(c)

FIGURE 5: (a) shows phase-contrast micrographs of untreated control hDMSCs which showed typical MSC-like phenotype. (b) shows long elongated tube-like cells seen on day 14 after hDMSCs were treated with $10 \mu \mathrm{M}$ of 5-azacytidine for 48 hours. (c) shows day 14 of hDMSCs treated with $20 \mu \mathrm{M}$-of 5-azacytidine for 48 hours. These cells showed cardiomyocyte-like cell elongations and cytoplasmic granularity.

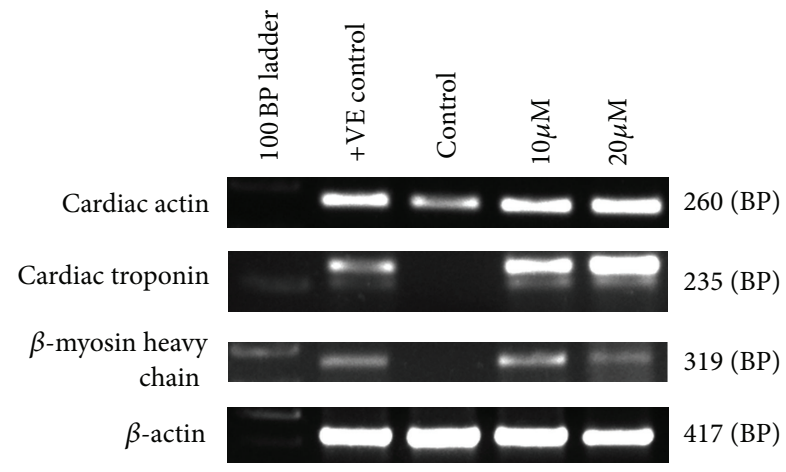

FIGURE 6: Gel electrophoresis analysis of cardiac specific genes of experiment set 1 shows that 5 -azacytidine treated $(10 \mu \mathrm{M}$ and $20 \mu \mathrm{M})$ hDMSCs were positive for $\alpha$-cardiac actin, $\beta$-myosin heavy chain, and cardiac troponin T, whereas untreated cells were positive for $\alpha$ cardiac actin and negative for $\beta$-myosin heavy chain, and cardiac troponin $\mathrm{T}$.

with 70\% ethanol for 1 hour and stained with Alizarin Red S stain (Fisher Scientifics) and incubated at room temperature for 30 minutes.

2.4. Cardiomyogenic Differentiation of Human Dermal Mesenchymal Stem Cells. To induce cardiac differentiation of hDMSCs, cells from passage 12 were treated with $10 \mu \mathrm{M}$ and $20 \mu \mathrm{M}$ with 5-azacytidine (Sigma-Aldrich Co.) for different time intervals. Three sets of experiments were carried out: 48 hours treatment, 4 days treatment, and 8 days treatment as outlined in Figure 1. For each experiment, around $1 \times 10^{4}$ cells of hDMSCs were seeded in $65 \mathrm{~mm}$ culture dishes (nunc) in three batches of which two were treated with $10 \mu \mathrm{M}$ and $20 \mu \mathrm{M}$ of 5-azacytidine, respectively, (on the second day after seeding) and the third batch was untreated and maintained in normal growth media as control group.

For the first experiment, hDMSCs were exposed to 5azacytidine in the growth medium for a short period of 48 hours after which cells were maintained in normal growth media (without 5-azacytidine) and the cells were allowed to differentiate over a period of 15 days. These cells were observed every day for any morphological changes. After 15 days, clear morphological changes were observed in the treated cells which were therefore fixed for light microscopy and gene expression studies.

Similarly, the second and third set of experiments involved treatment of hDMSCs for a period of 4 days and 8 days, respectively, after which the cells were sacrificed for molecular studies.

\subsection{Characterization of hDMSCs Cells Using Molecular Mark-} ers. Total RNA was extracted from treated and untreated hDMSCs cells as described above. RNA was extracted using TRIzol Reagent (Invitrogen) and quantified by using spectrophotometer (Eppendorf, Germany). The cDNA was synthesized using cDNA preparation kit using Random Hexamers as primers from Applied Biosystems. The protocol conditions were at $25^{\circ} \mathrm{C}$ for $10 \mathrm{~min}, 37^{\circ} \mathrm{C}$ for $120 \mathrm{~min}$, and terminated at $85^{\circ} \mathrm{C}$ for $5 \mathrm{~s}$. The synthesized cDNA was used to perform reverse transcriptase PCR to evaluate the expression of CD105, CD73, CD13, CD45, CD34, LIF, Oct4, Sox2, NANOG, and keratin 18, and beta-actin gene was used as a housekeeping gene to evaluate the efficiency of the reaction. The primer sequences and PCR conditions for the above described genes are referred from our previous studies [9, 12]. All primer sequences used for molecular marker study were checked for their respective product size by using BioEdit software which aligned to their original sequences from NCBI.

To determine the differentiation of hDMSCs to cardiomyocytes expression of cardiac-specific genes like $\alpha$-cardiac actin, cardiac troponin-T, and $\beta$-myosin heavy chain were analysed. The primer sequence for cardiac-specific genes has been mentioned in Table 1. The thermal profile for PCR was at $95^{\circ} \mathrm{C}$ for $5 \mathrm{~min}$ with denaturation at $94^{\circ} \mathrm{C}$ for $30 \mathrm{~s}$, annealing at $57^{\circ} \mathrm{C}$ for $30 \mathrm{~s}$ followed by a $1 \mathrm{~min}$ extension at $72^{\circ} \mathrm{C}$ and an additional 7 minute final extension after the completion of the last cycle. The PCR products were analysed using 2\% agarose 


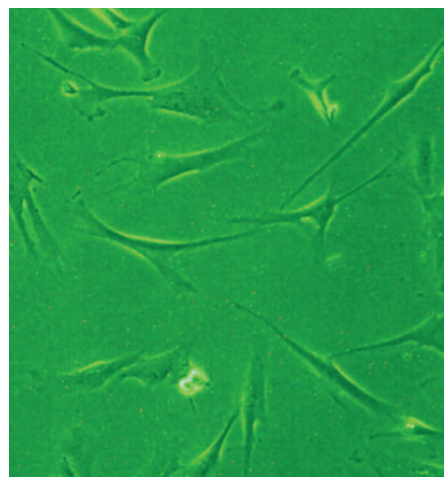

(a)

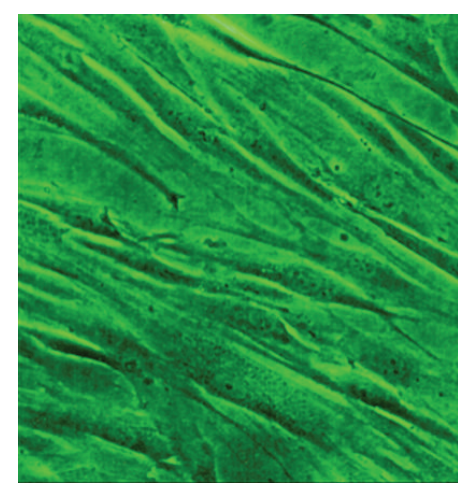

(b)

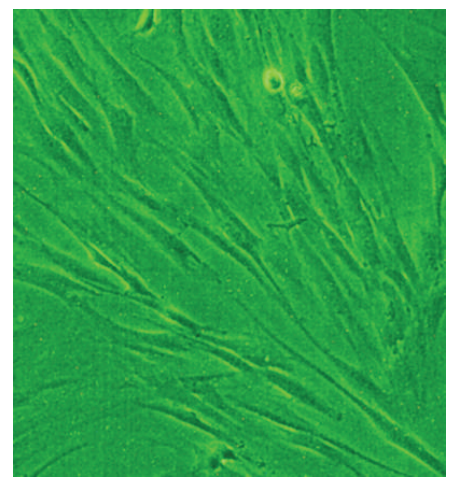

(c)

FIGURE 7: (a) shows phase-contrast micrographs of untreated control hDMSCs which showed no change in morphology. (b) shows stick-like appearance on day 4 , when hDMSCs were treated with $10 \mu \mathrm{M}$ of 5-azacytidine for 4 days. These cells became thinner and aligned parallel to each other. (c) shows cardiomyocyte-like appearance of hDMSCs treated with $20 \mu \mathrm{M}$-of 5 -azacytidine on day 4 . These cells are seen in groups of 6-7 cells.

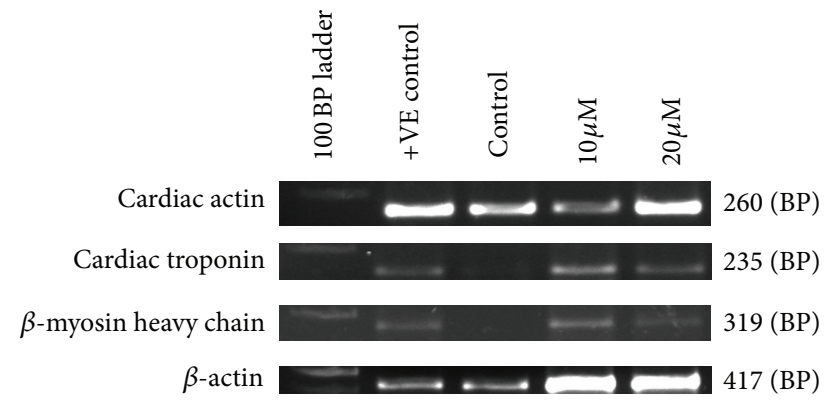

FIGURE 8: Gel electrophoresis analysis of PCR products of cardiac specific genes of experiment set 2 shows that 5 -azacytidine treated $(10 \mu \mathrm{M}$ and $20 \mu \mathrm{M})$ hDMSCs were positive for $\alpha$-cardiac actin, $\beta$ myosin heavy chain, and cardiac troponin $\mathrm{T}$, while untreated cells were positive for $\alpha$-cardiac actin and negative for $\beta$-myosin heavy chain and cardiac troponin $\mathrm{T}$.

gels for electrophoresis and image was taken under UV light (Cell Biosciences).

\section{Results}

3.1. Morphological Analysis of Human Dermal Mesenchymal Stem Cell Line. Figure 2(a) shows well-growing confluent cultures of hDMSCs cell line having spindle-like cells with distinct round nucleus and nuclei with scanty cytoplasmic granules giving it almost clear appearance. These cells grow longer with more or less smooth edges. Giemsa staining was performed to study the general morphology and cytoplasmic structure of the cell lines. The Giemsa stained cells show clear morphology of these cells with nucleus positioned in the centre as shown in Figure 2(b). Oil Red O staining showed that these cells were negative for Oil Red O staining, whereas this stain was taken up by the oil droplets secreted by these cells. Thus it was indicated that there is no spontaneous differentiation of these cells into adipocytes (Figure 2(c)).

3.2. Growth Rate Analysis of Human Dermal Mesenchymal Stem Cell Line. The plot of MTT assay (Figure 3) specifically shows the increase in cell proliferation with the progression of days (up to day 6). The doubling rate of dermal cells was observed to be around 36 hours.

3.3. Molecular Characterisation of Human Dermal Mesenchymal Stem Cell Line. To confirm that hDMSCs line isolated from explants maintained phenotype even at passage 12, they were checked for expression of genes specific for mesenchymal stem cells, hematopoietic stem cells and pluripotency. The hDMSCs showed expression of mesenchymal stem cells markers like CD105, CD73, and CD13. There was slight expression of CD34, a haematopoietic marker. However, CD45 was not expressed (data not shown).

Furthermore, to study the pluripotency of these cells, expressions of OCT-4, SOX-2, and NANOG were checked. The cultured cells express OCT-4, SOX-2, and NANOG suggesting that these cells have the potency to differentiate into other cell types when induced. These cells were also strongly positive for LIF expression thus indicating that these cells maintain themselves in an undifferentiated state. However, keratin-18 expression confirmed their epithelial nature. $\beta$-actin was used as a house-keeping gene.

The characterization studies show that human dermal mesenchymal stem Cell line developed from the human scalp punch biopsy is none other than mesenchymal stem cells which are multipotent and thus can be further utilised for differentiation studies.

\subsection{Differentiation of Human Dermal Mesenchymal Stem Cell Line into Cardiomyocytes}

3.4.1. Phase-Contrast Microscopy for hDMSCs after 48 Hours of 5-Azacytidine Treatment. In the first set of experiment, initially no changes in the morphology were observed. Slight elongations of cells were seen around day 3 and as time progressed cells started thinning out. It was only at the end of two weeks that very long and tube-like cells with dense granules in the cytoplasm and the nucleus slightly positioned 


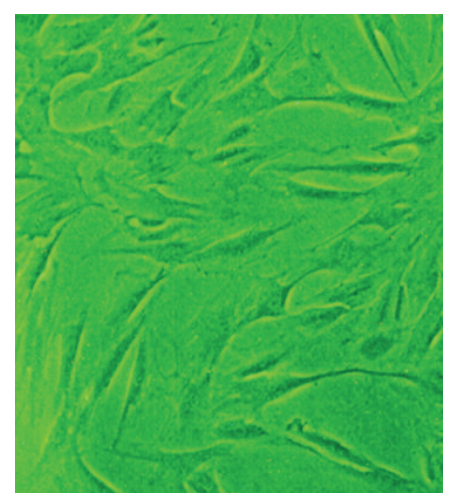

(a)

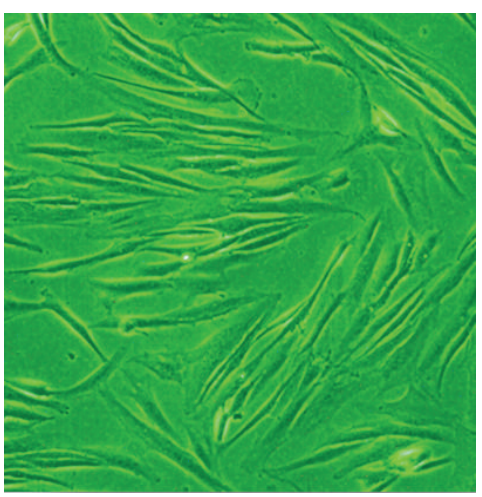

(b)

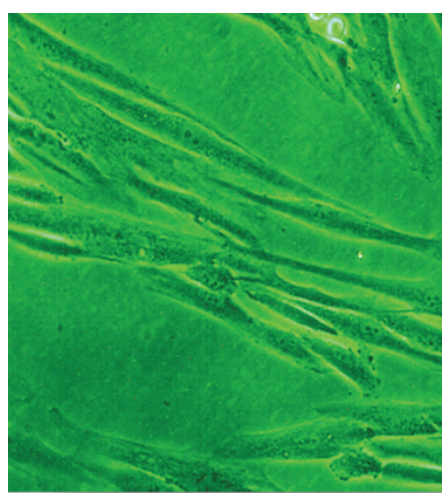

(c)

FIGURE 9: (a) Shows-phase contrast micrographs of untreated control hDMSCs with no changes in morphology. (b) shows cells on the 8th day showing stick-like morphology and cells were seen in groups. (c) shows cardiomyocyte-like appearance of hDMSCs treated with $20 \mu \mathrm{M}$ of 5-azacytidine on day 8 . These cells were seen in groups of 6-7 cells.

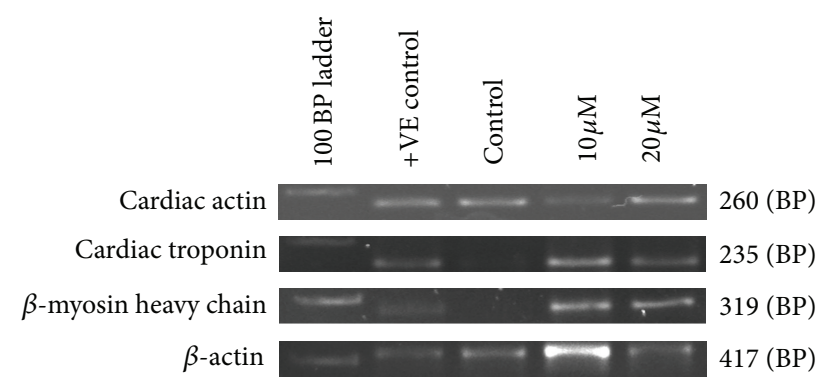

FIGURE 10: Gel electrophoresis analysis of cardiac-specific genes of experiment set 3 shows that 5 -azacytidine treated $(10 \mu \mathrm{M}$ and $20 \mu \mathrm{M}$ ) hDMSCs were positive for $\alpha$-cardiac actin, $\beta$-myosin heavy chain, and cardiac troponin $\mathrm{T}$ while untreated cells were positive for $\alpha$-cardiac actin and negative for $\beta$-myosin heavy chain and cardiac troponin $\mathrm{T}$.

away from the centre were seen as shown in Figures 5(a) and $5(\mathrm{~b})$.

3.4.2. Molecular Characterization of 48 Hour 5-Azacytidine Treated hDMSCs. The gene expression studies revealed that $\alpha$-cardiac actin was expressed by $10 \mu \mathrm{M}$ and $20 \mu \mathrm{M}$ of 5 azacytidine treated hDMSCs and control cells. The untreated cells were negative for cardiac troponin $\mathrm{T}$ and $\beta$-myosin heavy chain, whereas $10 \mu \mathrm{M}$ and $20 \mu \mathrm{M}$ of 5 -azacytidine treated hDMSCs cells showed positive expression of both these genes indicating that hDMSCs cells differentiated into cardiomyocytes.

There was not much variation between the $10 \mu \mathrm{M}$ and $20 \mu \mathrm{M} 5$-azacytidine treated hDMSCs in expression of these markers.

3.4.3. Phase-Contrast Microscopy for hDMSCs after 4 Days of 5-Azacytidine Treatment. These cells showed morphological changes from the third day after treatment. As compared to control dermal cells, treated cells became thinner and longer. By the third day, clusters of 4-5 cells were seen. These cells aligned parallel to each other in these clusters. By the fourth day, the cells show a peculiar stick-like appearance. This stick-like appearance and the parallel alignment gives the plate an overall striated pattern. These cells looked healthy and not much cell death was seen after treatment. Treated cells showed cardiomyocyte-like elongations as compared to control cells (MSCs). The cytoplasm seemed denser with granules as compared to untreated cell (Figure 7).

\subsubsection{Molecular Characterization of 4 Days 5-Azacytidine} Treated hDMSCs Cells. The molecular characterisation of $10 \mu \mathrm{M}$ and $20 \mu \mathrm{M} 5$-azacytidine treated hDMSCs revealed that these cells showed positive expression of cardiac specific genes $\alpha$-cardiac actin, cardiac troponin $\mathrm{T}$, and $\beta$-myosin heavy chain. The untreated hDMSCs showed positive expression of $\alpha$-cardiac actin but were negative for cardiac troponin $\mathrm{T}$ and $\beta$-myosin heavy chain. The gene expression analysis of the second set of experiment showed similar pattern of expression as compared to the first set (Figure 8); however, the intensity of expression is not as high as that seen in the 48 hours treated 5-azacytidine treated hDMSCs (Figure 6).

3.4.5. Phase-Contrast Microscopy for hDMSCs after 8 Days of 5-Azacytidine Treatment. Morphological changes were seen by the third day of induction in both $10 \mu \mathrm{M}$ and $20 \mu \mathrm{M} 5$ azacytidine treated hDMSCs. By the third and fourth day of treatment, the cells started becoming thinner as compared to the untreated cells and formed clusters of 6-7 cells aligned parallel to each other as shown in Figure 9(b). At higher magnification, the cytoplasm showed dense granules and a large nucleus as shown in Figure 9(c).

3.4.6. Molecular Characterization of 8 Days 5-Azacytidine Treated hDMSCs. As seen before, the gene expression analysis of the third set of experiment revealed an expression pattern similar to the second set. The $10 \mu \mathrm{M}$ and $20 \mu \mathrm{M}$ of 5 -azacytidine treated hDMSCs showed positive expression of $\alpha$-cardiac actin, cardiac troponin $\mathrm{T}$, and $\beta$-myosin heavy chain, whereas the untreated cells showed positive expression 


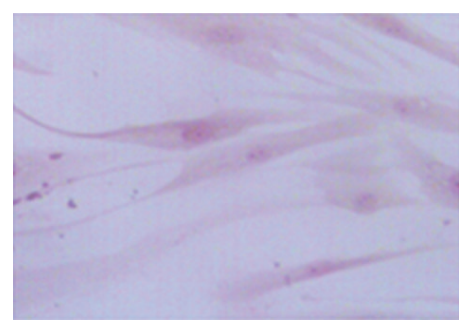

(a)

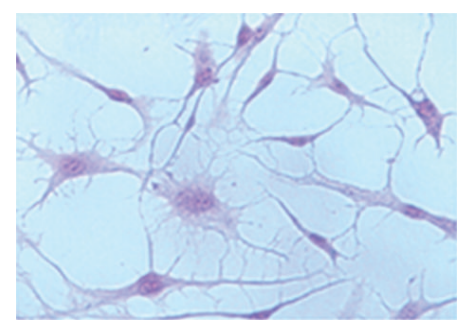

(b)

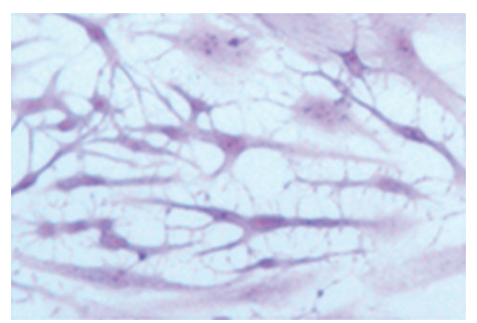

(c)

FIGURE 11: (a) shows light micrographs of Alizarin Red staining of control hDMSCs. No morphological changes seen in this group of cells. (b) shows light micrographs of Alizarin Red staining of $10 \mu \mathrm{M}$-5-azacytidine treated hDMSCs. These cells showed distinct web-like extensions in cytoplasm. (c) shows light micrographs of Alizarin Red staining of $20 \mu \mathrm{M} 5$-azacytidine treated hDMSCs. These cells showed web-like extensions in cytoplasm in greater frequencies as compared to $10 \mu \mathrm{M}$ 5-azacytidine treated hDMSCs.

of $\alpha$-cardiac actin and negative expression of cardiac troponin $\mathrm{T}$ and $\beta$-myosin (Figure 10); however, the intensities of bands were low as compared to 48 hours treated hDMSCs as shown in the Figure 6.

3.4.7. Calcium Deposition Analysis. Alizarin red is used in a biochemical assay to determine calcium deposited quantitatively by colorimetric analysis. In our study, we performed Alizarin Red staining to check for any change in mineral deposition in hDMSCs after being treated with 5-azacytidine. Clear calcium deposits were stained in cells treated with 5azacytidine as shown in Figure 11.

\section{Discussion}

Myocardial infarction causes a permanent damage to the heart which cannot be repaired in the adult human. The conventional line of treatment only relieves the symptoms but does not restore necrotic tissue $[1,2]$. Thus stem cellbased regenerative therapy could be one of the best available lifesaving options for patients suffering from MI. However, many questions remain unanswered about the mechanism of cardiac differentiation of MSCs in in vivo conditions and further studies are needed in this area of research [25]. Thus, we propose a safer and more appropriate line of treatment which includes isolation of dermal MSCs stem cells from patient's skin which can be differentiated into cardiomyocytes in vitro for regenerative therapies for MI, and thus providing patient-specific cardiomyocytes for cure of MI. Recently, Lior Gepstein from the Technion-Israel Institute of Technology has reported the use of human skin cells of elderly MI patients to make healthy beating heart tissue which can be used to treat this condition in near future [26].

Several research groups have reported that 5-azacytidine induces murine MSCs to differentiate into cardiomyocytes in vitro $[25,27]$. Similar work has been done by Liu and coworkers on rat bone marrow (BM) MSCs; however, this work has shown that only immortalized rat BMMSCs could differentiated into cardiomyocytes [21]. A recent study by Antonitsis et al. has shown that the adult human BMMSCs can differentiate into cardiomyocytes in vitro when treated with 5-azacytidine $(10 \mu \mathrm{M})$ [22]. Similarly, $\mathrm{Xu}$ et al. have shown that human BMMSCs could be isolated and used for repairing damaged heart [23]. Based on these studies, we have used our own developed hDMSCs cell line for differentiation of these cells into cardiomyocytes [24]. Before differentiation, we have reconfirmed their MSCs phenotypes by using MSCs molecular markers such as CD105, CD73, and CD13, and cell growth rate, pluripotency and differentiation characteristics of these cells which were prominently expressed even at passage 12 are shown in Figures 3 and 4. Therefore, we have used this cell line to differentiate into cardiomyocytes by 5 azacytidine.

Moreover, isolation of MSCs from bone marrow is an invasive procedure and therefore it is not a practical option to obtain samples from a patient so easily. On the other hand, human skin is one of the most abundant tissues which can be easily accessible anytime by a minimally invasive punch biopsy procedure. Thus, based on the present study, we recommend skin as a potential source of human adult MSCs which can differentiated into cardiomyocytes and can be used for regenerative therapeutic for MI.

Morphological analysis studies carried out by Antonitsis et al. have shown that the hMSCs showed multinucleation and cytoplasmic extensions after a week of treatment with 5 -azacytidine, followed by aggregation of approximately 20$30 \%$ of cells forming characteristic stick-like appearance and at four weeks these cells enlarged in size and connected with adjoining cells forming myotube-like structures [22]. In the present study, hDMSCs showed similar morphological changes within 3-4 days of induction when treated with 5azacytidine $(10 \mu \mathrm{M}$ and $20 \mu \mathrm{M})$ showing typical myotubelike structures confirming hDMSCs differentiation into cardiomyocytes. These cells appeared thinner giving them typical stick-like appearance and aligned parallel to each other forming connections. Small bunches of 4-5 parallel cells were observed and grew larger in size. Higher magnifications revealed that the cell cytoplasm became dense with granules and the position of the nucleus shifted slightly away from the centre, a typical feature also reported by the other studies $[22,23]$.

Several studies have reported the expression of cardiac specific markers like $\alpha$-cardiac actin, cardiac troponin T, and $\beta$-myosin Heavy Chain to confirm cardiomyocyte differentiation $[19,21-23]$. The gene expression analysis in the present 
study confirms that 5-azacytidine ( $10 \mu \mathrm{M}$ and $20 \mu \mathrm{M})$ treated hDMSCs can differentiate into cardiomyocytes. Cardiac actin monomers are principle components of the thin filament of cardiac muscle myofibrils and sarcomeres. The sarcomere is the basic structural element that mediates the contraction of cardiac muscle [28]. Cardiac troponin $\mathrm{T}$ is known to be the early marker of myogenic differentiation [23]. It is a protein essential for calcium-regulated myofibrillar ATPase activity and is expressed in the human heart and is required for muscle contractile movement [29]. In our study, Alizarin Red staining has shown several calcium granules in the cytoplasm of differentiated cardiomyocytes, thus indicating that the cells are active and may help to improve muscle contractile movement in MI patients. Similarly, by molecular studies, it was clearly shown that 5 -azacytidine treated cell expressed cardiac troponin $\mathrm{T}$ as shown in Figures 6, 8, and 10. $\beta$-myosin heavy chain, a member of the muscle myosin family, is one of the subunit proteins encoded by the gene. $\beta$-myosin heavy chain is expressed predominantly in normal human ventricle [30]. In the present study, $\beta$-myosin heavy chain expression was seen in differentiated cardiomyocytes indicating that these cells may be useful in repairing damage to heart ventricle.

Differentiation of hDMSCs into cardiomyocytes mainly depends on the treatment protocol followed. In the present study, it was observed that the cells which were exposed to $48 \mathrm{hr}$ of treatment and allowed to differentiate over a period of 15 days have shown better expression of $\alpha$-cardiac actin, cardiac troponin $\mathrm{T}$, and $\beta$-myosin heavy chain indicating that this protocol is more feasible for differentiation of functional cardiomyocytes than treatment for an extended period of 4 days and 8 days as shown in experiment 2 and 3, respectively.

\section{Conclusions}

This study suggests that as skin is the most abundant and easily accessible tissue from a patient, it will be easier to develop hDMSCs cell line which can differentiated into cardiomyocytes for the treatment of MI patients in a span of 2-3 months. Thus our method puts forth a safer and minimally invasive technique to provide relief for patients suffering from coronary disorders like MI. However, further studies have to be carried out before they are clinically applied to MI patients.

\section{Conflict of Interests}

The authors declare that they have no conflict of interests.

\section{Acknowledgments}

The authors wish to thank the management of Jaslok Hospital and Research Centre, Mumbai, India, for sanctioning and providing financial assistance for stem cell research, project no. $491 \mathrm{~A} / \mathrm{c} .27814$.

\section{References}

[1] Y. S. Yoon, N. Lee, and H. Scadova, "Myocardial regeneration with bone-marrow-derived stem cells," Biology of the Cell, vol. 97, no. 4, pp. 253-263, 2005.
[2] H. M. Nugent and E. R. Edelman, “Tissue engineering therapy for cardiovascular disease," Circulation Research, vol. 92, no. 10, pp. 1068-1078, 2003.

[3] L. Reinlib and L. Field, "Cell transplantation as future therapy for cardiovascular disease?: a workshop of the National Heart, Lung, and Blood Institute," Circulation, vol. 101, no. 18, pp. e182e187, 2000.

[4] J. J. Minguell, A. Erices, and P. Conget, "Mesenchymal stem cells," Experimental Biology and Medicine, vol. 226, no. 6, pp. 507-520, 2001.

[5] E. A. Jones, S. E. Kinsey, A. English et al., "Isolation and characterization of bone marrow multipotential mesenchymal progenitor cells," Arthritis and Rheumatism, vol. 46, no. 12, pp. 3349-3360, 2002.

[6] L. L. Lu, Y. J. Liu, S. G. Yang et al., "Isolation and characterization of human umbilical cord mesenchymal stem cells with hematopoiesis-supportive function and other potentials," Haematologica, vol. 91, no. 8, pp. 1017-1026, 2006.

[7] O. K. Lee, T. K. Kuo, W. M. Chen, K. D. Lee, S. L. Hsieh, and T. H. Chen, "Isolation of multipotent mesenchymal stem cells from umbilical cord blood," Blood, vol. 103, no. 5, pp. 1669-1675, 2004.

[8] J. Suchánek, T. Soukup, R. Ivancaková et al., "Human dental pulp stem cells-isolation and long term cultivation," Acta Medica, vol. 50, no. 3, pp. 195-201, 2007.

[9] P. D. Potdar and J. P. Sutar, "Establishment and molecular characterization of mesenchymal stem cell lines derived from human visceral \& subcutaneous adipose tissues," Journal of Stem Cells and Regenerative Medicine, vol. 6, no. 1, pp. 26-35, 2010.

[10] P. D. Potdar and R. P. Subedi, "Defining molecular phenotypes of mesenchymal and hematopoietic stem cells derived from peripheral blood of acute lymphocytic leukemia patients for regenerative stem cell therapy," Journal of Stem Cells \& Regenerative Medicine, vol. 7, pp. 1-10, 2011.

[11] P. D. Potdar and S. B. D'souza, "Isolation of Oct $4^{+}, \mathrm{Nanog}^{+}$and SOX2 $2^{-}$mesenchymal cells from peripheral blood of a diabetes mellitus patient," Human Cell, vol. 24, no. 1, pp. 51-55, 2011.

[12] P. D. Potdar and S. Chougule, "Establishment and molecular characterization of breast cancer mesenchymal stem cell line derived from human non-metastasis breast cancer tumor," Stem Cell Discovery, vol. 1, no. 2, pp. 21-28, 2011.

[13] I. Sekiya, B. L. Larson, J. T. Vuoristo, J. G. Cui, and D. J. Prockop, "Adipogenic differentiation of human adult stem cells from bone marrow stroma (MSCs)," Journal of Bone and Mineral Research, vol. 19, no. 2, pp. 256-264, 2004.

[14] B. C. Heng, T. Cao, and E. H. Lee, "Directing stem cell differentiation into the chondrogenic lineage in vitro," Stem Cells, vol. 22, no. 7, pp. 1152-1167, 2004.

[15] K. S. Park, Y. S. Lee, and K. S. Kang, "In vitro neuronal and osteogenic differentiation of mesenchymal stem cells from human umbilical cord blood," Journal of Veterinary Science, vol. 7, no. 4, pp. 343-348, 2006.

[16] J. Oswald, S. Boxberger, B. Jørgensen et al., "Mesenchymal stem cells can be differentiated into endothelial cells in vitro," Stem Cells, vol. 22, no. 3, pp. 377-384, 2004.

[17] K. D. Lee, T. K. C. Kuo, J. Whang-Peng, and Y. F. Chung, "In vitro hepatic differentiation of human mesenchymal stem cells," Hepatology, vol. 40, no. 6, pp. 1275-1284, 2004.

[18] D. Woodbury, E. J. Schwarz, D. J. Prockopand, and I. B. Black, "Adult rat and human bone marrow stromal cells differentiate into neurons," Journal of Neuroscience Research, vol. 61, pp. 364370, 2000. 
[19] S. Makino, K. Fukuda, S. Miyoshi et al., "Cardiomyocytes can be generated from marrow stromal cells in vitro," The Journal of Clinical Investigation, vol. 103, no. 5, pp. 697-705, 1999.

[20] M. Shi, Z. W. Liu, and F. S. Wang, "Immunomodulatory properties and therapeutic application of mesenchymal stem cells," Clinical and Experimental Immunology, vol. 164, no. 1, pp. 1-8, 2011.

[21] Y. Liu, J. Song, W. Liu, Y. Wan, X. Chen, and C. Hu, "Growth and differentiation of rat bone marrow stromal cells: does 5-azacytidine trigger their cardiomyogenic differentiation?" Cardiovascular Research, vol. 58, no. 2, pp. 460-468, 2003.

[22] P. Antonitsis, E. Ioannidou-Papagiannaki, A. Kaidoglou, and C. Papakonstantinou, "In vitro cardiomyogenic differentiation of adult human bone marrow mesenchymal stem cells. The role of 5-azacytidine," Interactive Cardiovascular and Thoracic Surgery, vol. 6, no. 5, pp. 593-597, 2007.

[23] W. Xu, X. Zhang, H. Qian et al., "Mesenchymal stem cells from adult human bone marrow differentiate into a cardiomyocyte phenotype in vitro," Experimental Biology and Medicine, vol. 229, no. 7, pp. 623-631, 2004.

[24] P. Potdar and K. Dand Kumar, "Establishment and molecular characterisation of human dermal mesenchymal like stem cells derived from human scalp biopsy of androgenetic alopecia patient," Stem cell Discovery. In press.

[25] D. Orlic, J. Kajstura, S. Chimenti et al., "Bone marrow cells regenerate infarcted myocardium," Nature, vol. 410, no. 6829, pp. 701-705, 2001.

[26] L. Zwi-Dantsis, I. Huber, M. Habib et al., "Derivation and cardiomyocyte differentiation of induced pluripotent stem cells from heart failure patients," European Heart Journal, vol. 10, p. 1093, 2012.

[27] F. P. Barry and J. M. Murphy, "Mesenchymal stem cells: clinical applications and biological characterization," The International Journal of Biochemistry \& Cell Biology, vol. 36, no. 4, pp. 568584, 2004.

[28] S. Ebashi, "Ca ${ }^{2+}$ and the contractile proteins," Journal of Molecular and Cellular Cardiology, vol. 16, no. 2, pp. 129-136, 1984.

[29] A. S. Zot and J. D. Potter, "Structural aspects of troponintropomyosin regulation of skeletal muscle contraction," Annual Review of Biophysics and Biophysical Chemistry, vol. 16, pp. 535$559,1987$.

[30] A. Matsakas, "Molecular advances shed light on cardiac myosin heavy chain expression in health and disease," Experimental Physiology, vol. 94, no. 12, pp. 1161-1162, 2009. 


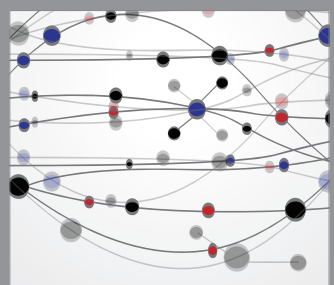

The Scientific World Journal
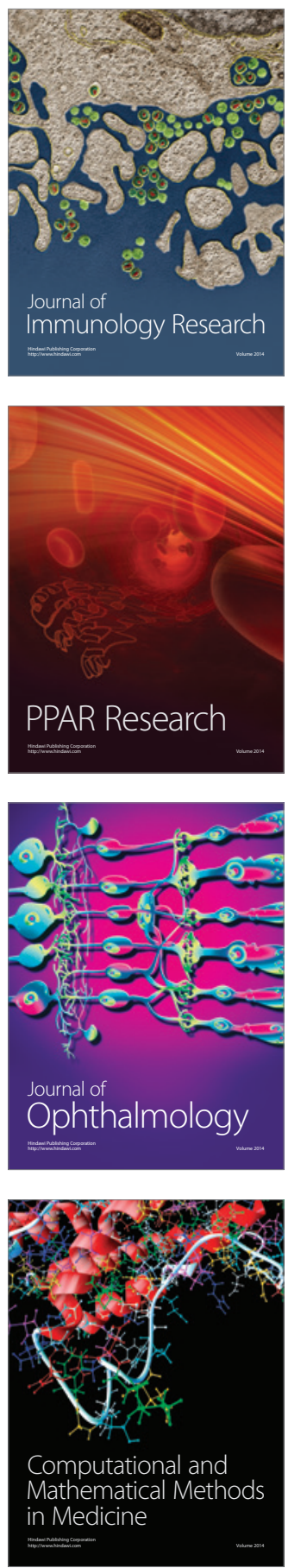

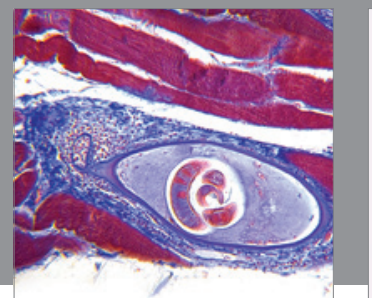

Gastroenterology

Research and Practice
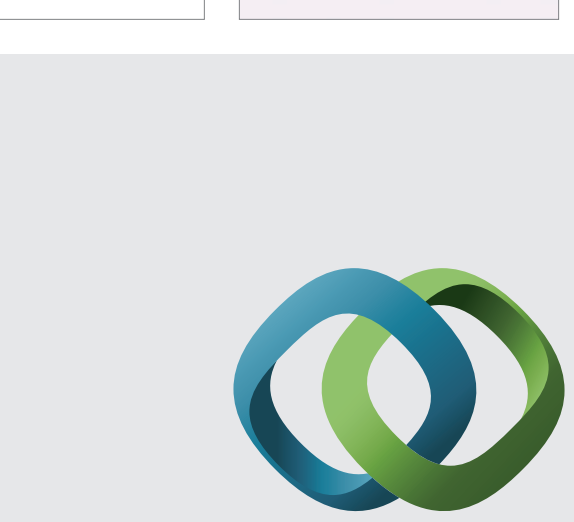

\section{Hindawi}

Submit your manuscripts at

http://www.hindawi.com
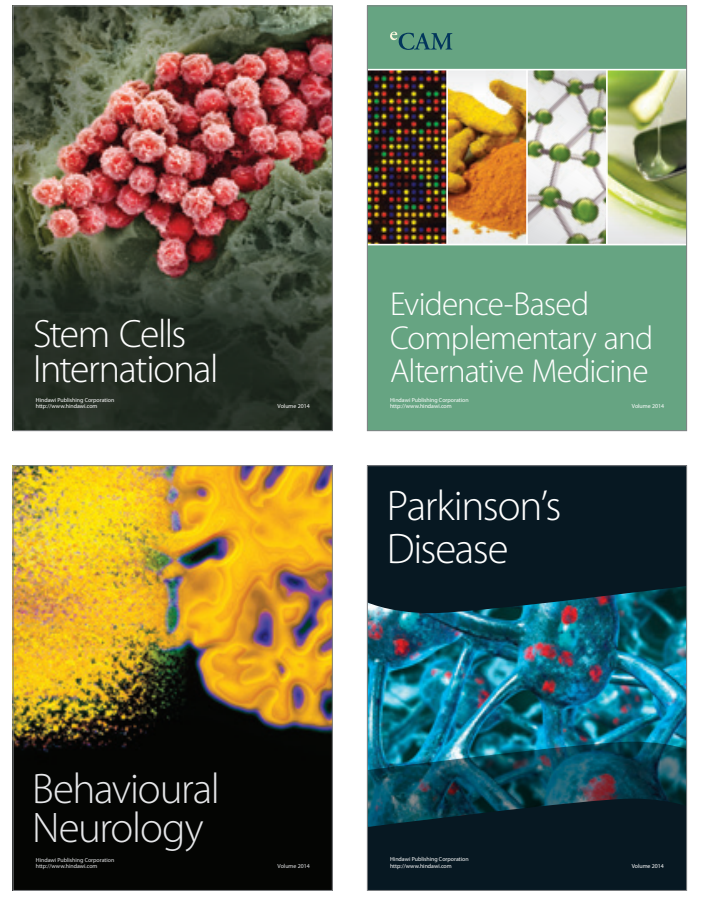
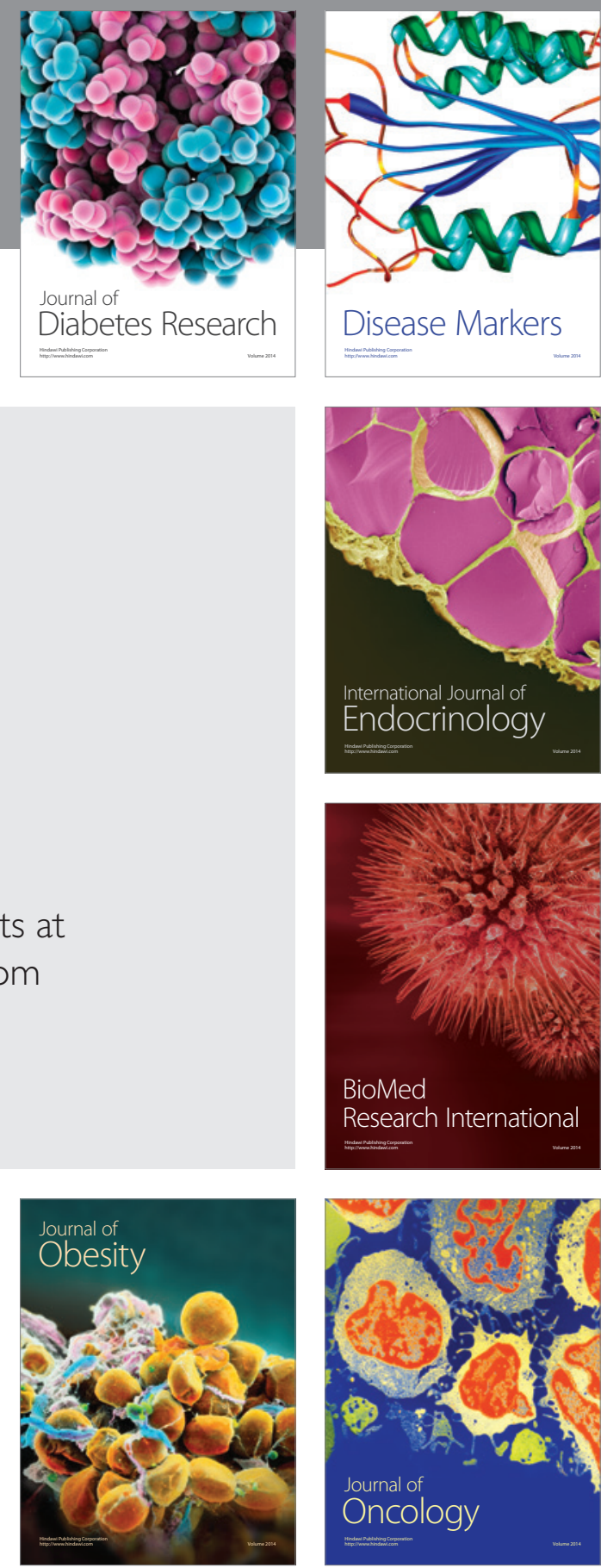

Disease Markers
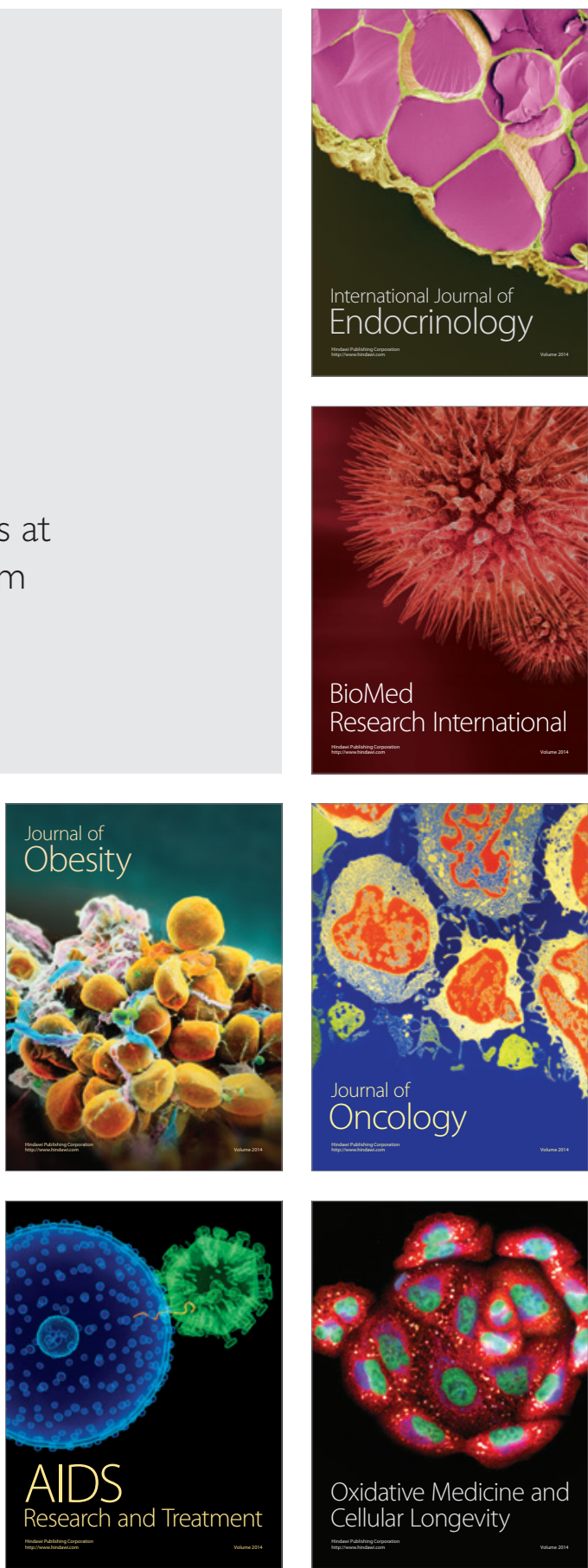